MATHEMATICS OF COMPUTATION

Volume 76, Number 259, July 2007, Pages 1449-1468

S 0025-5718(07)01918-7

Article electronically published on January 31,2007

\title{
NUMERICALLY SATISFACTORY SOLUTIONS OF HYPERGEOMETRIC RECURSIONS
}

\author{
AMPARO GIL, JAVIER SEGURA, AND NICO M. TEMME
}

Abstract. Each family of Gauss hypergeometric functions

$$
f_{n}={ }_{2} F_{1}\left(a+\varepsilon_{1} n, b+\varepsilon_{2} n ; c+\varepsilon_{3} n ; z\right), n \in \mathbb{Z},
$$

for fixed $\varepsilon_{j}=0, \pm 1$ (not all $\varepsilon_{j}$ equal to zero) satisfies a second order linear difference equation of the form

$$
A_{n} f_{n-1}+B_{n} f_{n}+C_{n} f_{n+1}=0 .
$$

Because of symmetry relations and functional relations for the Gauss functions, many of the 26 cases (for different $\varepsilon_{j}$ values) can be transformed into each other. In this way, only with four basic difference equations can all other cases be obtained. For each of these recurrences, we give pairs of numerically satisfactory solutions in the regions in the complex plane where $\left|t_{1}\right| \neq\left|t_{2}\right|, t_{1}$ and $t_{2}$ being the roots of the characteristic equation.

\section{INTRODUCTION}

The families of Gauss hypergeometric functions

$$
y_{n}={ }_{2} F_{1}\left(\begin{array}{c}
a+\varepsilon_{1} n, b+\varepsilon_{2} n \\
c+\varepsilon_{3} n
\end{array} ; z\right), \quad n \in \mathbb{Z},
$$

where

$$
{ }_{2} F_{1}\left(\begin{array}{c}
a, b \\
c
\end{array} ; z\right)=\sum_{n=0}^{\infty} \frac{(a)_{n}(b)_{n}}{(c)_{n} n !} z^{n}, \quad|z|<1,
$$

satisfy second order linear difference equations (three-term recurrence relations) of the form

$$
A_{n} y_{n-1}+B_{n} y_{n}+C_{n} y_{n+1}=0 .
$$

Here, $\varepsilon_{j} \in \mathbb{Z}$ are fixed and not all $\varepsilon_{j}$ are equal to zero.

In this paper, we consider the cases $\epsilon_{j}=0, \pm 1$. That these recurrences exist and can be obtained from relations between contiguous functions (see [1, pp. 557-558]) are well-known facts. However, the condition of these recurrences is essentially an unexplored issue.

For a numerical use of a recurrence relation, it is of crucial importance to know whether a recurrence admits a minimal solution and to identify such a solution

Received by the editor October 18, 2005 and, in revised form, February 2, 2006.

2000 Mathematics Subject Classification. Primary 33C05, 39A11, 41A60, 65D20.

Key words and phrases. Gauss hypergeometric functions, recursion relations, difference equations, stability of recursion relations, numerical evaluation of special functions, asymptotic analysis.

(C)2007 American Mathematical Society Reverts to public domain 28 years from publication 
when it exists. A solution $f_{n}$ of a three-term recurrence relation (TTRR) is said to be minimal as $n \rightarrow+\infty$ when, for any other solution independent of $f_{n}, g_{n}$, $\lim _{n \rightarrow+\infty} f_{n} / g_{n}=0 ; g_{n}$ is said to be a dominant solution. The minimal solution is unique up to a constant factor (not depending on $n$ ). The computation of values of $f_{n}$ for large $n$ by a forward (increasing $n$ ) application of the TTRR is a bad conditioned process, while the backward application of the recurrence is generally well conditioned. The opposite happens for the dominant solutions.

When a TTRR admits a minimal solution, an independent pair of solutions of this TTRR $\left\{f_{n}, g_{n}\right\}$ is said to be numerically satisfactory for large $n$ when it includes the minimal solution. The goal of this article is to obtain numerically satisfactory solutions for the hypergeometric recursions in the complex plane.

As shown in [3], of the 26 possible recursions (when $\left|\epsilon_{j}\right| \leq 1$ ) in principle only five have to be studied, because of symmetry relations and connection formulas. That is, because of the relations

$$
\begin{aligned}
{ }_{2} F_{1}\left(\begin{array}{c}
a, b \\
c
\end{array} ;\right) & ={ }_{2} F_{1}\left(\begin{array}{c}
b, a \\
c
\end{array} ; z\right), \\
{ }_{2} F_{1}\left(\begin{array}{c}
a, b \\
c
\end{array}\right) & =(1-z)^{-a}{ }_{2} F_{1}\left(\begin{array}{cc}
a, c-b & ; \\
c & z-1
\end{array}\right), \\
{ }_{2} F_{1}\left(\begin{array}{c}
a, b \\
c
\end{array}\right) & =(1-z)^{-b}{ }_{2} F_{1}\left(\begin{array}{c}
c-a, b \\
c
\end{array} \frac{z}{z-1}\right), \\
{ }_{2} F_{1}\left(\begin{array}{c}
a, b \\
c
\end{array}\right) & =(1-z)^{c-a-b}{ }_{2} F_{1}\left(\begin{array}{c}
c-a, c-b \\
c
\end{array}\right) .
\end{aligned}
$$

Using these relations, it follows that we need to study the recursions for the following five basic forms [3]:

$$
\begin{aligned}
& { }_{2} F_{1}\left(\begin{array}{c}
a+n, b+n \\
c
\end{array} ; z\right), \quad{ }_{2} F_{1}\left(\begin{array}{c}
a+n, b+n \\
c-n
\end{array} ;\right), \quad{ }_{2} F_{1}\left(\begin{array}{c}
a+n, b \\
c
\end{array} z\right), \\
& { }_{2} F_{1}\left(\begin{array}{c}
a+n, b \\
c-n
\end{array} ; z\right), \quad{ }_{2} F_{1}\left(\begin{array}{c}
a, b \\
c+n
\end{array} ; z\right) .
\end{aligned}
$$

However, as we will see when explicitly building solutions for these recurrences, the third and the last cases in Eq. (1.5) are also related, and only four recurrences need to be studied.

In $[3$ we have described the domains in the complex $z$-plane where minimal and dominant solutions of the difference equations have to be determined. In this paper, we determine the minimal solutions in each of these domains which, together with any dominant solution, forms a numerically satisfactory pair of solutions of the corresponding three term recurrence relation.

In order to find the minimal solutions in each domain, it will be important to build solutions of the recurrence relations based on expansions around the three singular regular points of the differential equation: $z=0,1, \infty$. The following set of functions, solutions of the Gauss hypergeometric equation, provides the starting 
point for finding such solutions of the TTRR:

$$
\begin{aligned}
& w_{1}={ }_{2} F_{1}\left(\begin{array}{c}
a, b \\
c
\end{array}\right) \\
& w_{2}=z^{1-c_{2} F_{1}}\left(\begin{array}{c}
1+a-c, 1+b-c \\
2-c
\end{array} z\right) \\
& w_{3}={ }_{2} F_{1}\left(\begin{array}{c}
a, b \quad ; 1-z \\
a+b+1-c
\end{array}\right) \\
& w_{4}=(1-z)^{c-a-b}{ }_{2} F_{1}\left(\begin{array}{c}
c-a, c-b \\
c+1-a-b
\end{array} ; 1-z\right), \\
& w_{5}=\left(z^{-1} e^{i \pi}\right)^{a}{ }_{2} F_{1}\left(\begin{array}{c}
a, a+1-c \\
a+1-b
\end{array} ; \frac{1}{z}\right) \\
& w_{6}=\left(z^{-1} e^{i \pi}\right)_{2}^{b} F_{1}\left(\begin{array}{c}
b, b+1-c \\
b+1-a
\end{array} ; \frac{1}{z}\right) .
\end{aligned}
$$

This set of solutions plays an important role in defining pairs of linearly independent solutions of the hypergeometric differential equation. With the relations in (1.4) Kummer's 24 solutions of the differential equation can be given; see [4, p. 67]. Each element of the 24 solutions can be written as a linear combination of two other elements.

The six functions in (1.6) are also important in the theory of the difference equations for the Gauss hypergeometric function. When any of these functions satisfies a second order recursion relation, the remaining five functions $w_{j}$ satisfy the same relation provided we multiply each of these solutions by an appropriate factor. These factors follow from the linear relations between the functions $w_{j}$.

For our purposes we mention four relations (see [4, pp. 70-71]) in which $w_{1}$ is written in terms of the other $w_{j}, j=2,3,4,5,6$. There are six more relations, but we need only 4 relations. These are

$$
\begin{aligned}
w_{1} & =\frac{\Gamma(a+1-c) \Gamma(b+1-c)}{\Gamma(1-c)}\left[\frac{w_{3}}{\Gamma(a+b+1-c)}-\frac{\Gamma(c-1)}{\Gamma(a) \Gamma(b)} w_{2}\right] \\
& =\frac{\Gamma(1-a) \Gamma(1-b)}{\Gamma(1-c)}\left[\frac{w_{4}}{\Gamma(c+1-a-b)}-\frac{\Gamma(c-1)}{\Gamma(c-a) \Gamma(c-b)} w_{2}\right] \\
& =\frac{\Gamma(1-b) \Gamma(1+a-c)}{\Gamma(1-c)}\left[\frac{w_{5}}{\Gamma(1+a-b)}-\frac{\Gamma(c-1) e^{i \pi(c-1)}}{\Gamma(a) \Gamma(c-b)} w_{2}\right] \\
& =\frac{\Gamma(1-a) \Gamma(1+b-c)}{\Gamma(1-c)}\left[\frac{w_{6}}{\Gamma(1+b-a)}-\frac{\Gamma(c-1) e^{i \pi(c-1)}}{\Gamma(b) \Gamma(c-a)} w_{2}\right] .
\end{aligned}
$$

When, for example the function $w_{1}$ of (1.6) is in the form ${ }_{2} \mathrm{~F}_{1}(a+n, b+n ; c ; z)$ and satisfies a certain three-term recursion relation, any of the $w_{j}$ at the right-hand sides of (1.7) (with $a$ and $b$ replaced by $a+n$ and $b+n$, and with the given ratios of gamma functions) are seen to satisfy the same recursion relation. This can also be checked by direct substitution. 
We will use these relations to build 6 different solutions for each recurrence relation. Of course, only two solutions will be independent but, as we will see, one of them will be minimal when the roots of the characteristic equation have different moduli.

When building the six solutions, when necessary we will also use the relation

$$
\Gamma(p-n)=\frac{(-1)^{n} \pi}{\sin (\pi p) \Gamma(n+1-p)}
$$

in order to avoid front factors with gamma functions of the form $\Gamma(p-n)$, for $p$ any combination of the $a, b, c$ parameters. Also, the relations (1.4) will be used to express some of the solutions in a more symmetric form. Finally, we will neglect all front factors not depending on $n$.

In the following sections we give the unique minimal solution (up to a multiplicative constant) and five dominant solutions for each recursion relation corresponding with the 5 basic forms in (1.5). Each recursion relation has one or more different domains in the complex $z$-plane for which the minimal and dominant solutions have to be given.

\section{Minimal AND DOMinant SOlutions}

Consider the difference equation (1.3) and define

$$
\alpha_{n}:=\frac{A_{n}}{C_{n}}, \quad \beta_{n}:=\frac{B_{n}}{C_{n}} .
$$

Then for all equations considered in this paper $\alpha_{n}$ and $\beta_{n}$ tend to well-defined limits (except, perhaps, at the singular points of the hypergeometric differential equation). We write

$$
\alpha:=\lim _{n \rightarrow \infty} \alpha_{n}, \quad \beta:=\lim _{n \rightarrow \infty} \beta_{n} .
$$

Perron's theorem (see [6, Appendix B]) gives in the case of finite limits the following result, which is also known as Poincaré's theorem.

Theorem 1 (Poincaré). Let $t_{1}$ and $t_{2}$ denote the zeros of the characteristic equation $t^{2}+\beta t+\alpha=0$. Then, if $\left|t_{1}\right| \neq\left|t_{2}\right|$ the difference equation (1.3) has two linear independent solutions $f_{n}$ and $g_{n}$ with the properties

$$
\lim _{n \rightarrow+\infty} \frac{f_{n}}{f_{n-1}}=t_{1}, \quad \lim _{n \rightarrow+\infty} \frac{g_{n}}{g_{n-1}}=t_{2} .
$$

If $\left|t_{1}\right|=\left|t_{2}\right|$, then

$$
\limsup _{n \rightarrow \infty}\left|y_{n}\right|^{\frac{1}{n}}=\left|t_{1}\right|
$$

for any non-trivial solution $y_{n}$ of (1.3).

When $\left|t_{1}\right| \neq\left|t_{2}\right|$ the minimal solution is the one whose ratio $\left(f_{n} / f_{n-1}\right.$ or $\left.g_{n} / g_{n-1}\right)$ converges to the root with the smallest modulus. When $\left|t_{1}\right|=\left|t_{2}\right|$ the theorem is inconclusive with respect to the existence of a minimal solution.

The same type of analysis can be considered as $n \rightarrow-\infty$. By defining $\hat{y}_{n}=y_{-n}$, we can use Theorem 1 for studying the behaviour of $\hat{y}_{n}$ and $n \rightarrow+\infty$. Because for the hypergeometric case $\lim _{n \rightarrow \pm \infty} \alpha_{n}=\alpha$ and $\lim _{n \rightarrow \pm \infty} \beta_{n}=\beta$ the characteristic equation for $\hat{y}_{n}$ turns out to be reciprocal to the previous case, with roots $1 / t_{1}$ and $1 / t_{2}$. A simple way to analyze the behaviour as $n \rightarrow-\infty$ is the following. 
Theorem 2. Suppose that $\lim _{n \rightarrow \pm \infty} \alpha_{n}=\alpha$ and $\lim _{n \rightarrow \pm \infty} \beta_{n}=\beta$, and $t_{1}$ and $t_{2}$ are the roots of $t^{2}+\beta t+\alpha=0$. Let us assume that, for instance, $\left|t_{1}\right|<\left|t_{2}\right|$.

Then the difference equation has a minimal solution both as $n \rightarrow+\infty$ and as $n \rightarrow-\infty$.

The minimal solution when $n \rightarrow+\infty$ satifies

$$
\lim _{n \rightarrow+\infty} \frac{y_{n}}{y_{n-1}}=t_{1}
$$

while the minimal solution as $n \rightarrow-\infty$ is such that

$$
\lim _{n \rightarrow-\infty} \frac{y_{n}}{y_{n-1}}=t_{2} .
$$

We will use this theorem for identifying the minimal solutions in each region in the complex plane for which $\left|t_{1}\right| \neq\left|t_{2}\right|$. For this, we first need to determine solutions of the recurrence and then to obtain the asymptotic behaviour of ratios of these solutions as $n \rightarrow \pm \infty$.

In [3] we announced that all information on minimal and dominant solutions, for all recursions and for all domains, could be obtained by using asymptotic estimates of the Gauss hypergeometric functions for large parameters, as given in [5]; some of these estimates are given in [4, 235-242]. Fortunately, the study becomes more straightforward by considering the asymptotics of the ratios $y_{n} / y_{n-1}$, which is the only information needed in Theorems 1 and 2 . In addition, the study becomes much more simple by taking into account the following result:

Theorem 3. The character of the solutions of the hypergeometric TTRR cannot change in a connected domain where the characteristic roots $t_{1}, t_{2}$ satisfy $\left|t_{1}\right| \neq\left|t_{2}\right|$ for all $z$ in the domain.

This, together with the different behaviour of the roots $t_{1}$ and $t_{2}$ near the singular points of the hypergeometric $\operatorname{ODE}(0,1, \infty)$ allows for a simple identification by studying the behaviour of the solutions around these points.

For proving Theorem 3 we only need to prove that the solutions of the recurrence relation, $y_{n}$, are such that the ratio

$$
H_{n}=y_{n} / y_{n-1}
$$

has, for fixed $z$, a bounded derivative as $n \rightarrow \infty$. Indeed, because for each $z$, these ratios converge to one of the roots of the characteristic equation, when there exists $C$ such that $\left|H_{n}^{\prime}(z)\right|<C$ for large $n$ (and this is true for each of the six solutions we provide for each recurrence), we can guarantee that the limit may only change (from $t_{1}$ to $t_{2}$ ) when crossing the curves $\left|t_{1}\right|=\left|t_{2}\right|$ for any of the solutions.

A first step for proving the boundedness of the derivative is the following result:

Theorem 4. Under the conditions of Theorem 1 with $\alpha \neq 0$, let $\left\{y_{k}\right\}, k \in \mathbb{N}$, be a solution of the hypergeometric recurrence $y_{n+1}+\beta_{n} y_{n}+\alpha_{n} y_{n-1}=0$ and let $\left|t_{1}\right| \neq\left|t_{2}\right|$ (and therefore $\beta \neq 0$ and $\beta^{2}-4 \alpha \neq 0$ ). Then

$$
H_{n} \equiv \frac{y_{n}}{y_{n-1}}=t\left(1+h_{n}\right)
$$

where $t$ is a root of the characteristic equation and $h_{n}=\mathcal{O}(1 / n)$ as $n \rightarrow \infty$. 
Proof. From Poincaré's theorem, we know that $H_{n}=t\left(1+h_{n}\right)$ where $h_{n} \rightarrow 0$. In addition, because $\beta_{n}$ and $\alpha_{n}$ are rational functions of $n$ with finite limits as $n \rightarrow \infty$, then $\beta_{n}=\beta\left(1+\gamma_{n}\right)$ and $\alpha_{n}=\alpha\left(1+\delta_{n}\right)$ with $\delta_{n}, \gamma_{n}=\mathcal{O}(1 / n)$. Substituting these estimates into the difference equation satisfied by $H_{n}$ :

$$
\alpha_{n}+\beta_{n} H_{n}+H_{n} H_{n+1}=0,
$$

and using the characteristic equation, we have:

$$
\beta t\left(\gamma_{n}-h_{n+1}\right)\left(1+h_{n}\right)+\alpha\left(\delta_{n}-h_{n}-h_{n+1}\left(1+h_{n}\right)\right)=0 .
$$

Now, supposing that $h_{n}$ goes to zero slower that $1 / n$, we can write:

$$
\beta t h_{n+1}+\alpha\left(h_{n+1}+h_{n}\right)+o\left(h_{n}\right)=0
$$

therefore $\beta t+\alpha \neq 0$ and we can write:

$$
\lim _{n \rightarrow \infty} \frac{h_{n+1}}{h_{n}}=-\frac{\alpha}{\alpha+\beta t} \equiv K .
$$

But this is in contradiction with the hypothesis, for all values of $K$. Indeed, if $|K|<1$, then $h_{n}$ goes to zero as $K^{n}$ (faster that $1 / n$ ) while, if $|K|>1, h_{n}$ cannot go to zero; finally, if $|K|=1$, then $|\alpha|=|\alpha+\beta t|$, but because $\alpha+\beta t+t^{2}=0$ then $|t|^{2}=|\alpha|$; this is in contradiction with the fact that $\left|t_{1}\right| \neq\left|t_{2}\right|$, because $t$ is either $t_{1}$ or $t_{2}$ and $\alpha=t_{1} t_{2}$.

Now we can prove the boundedness of the derivative of $H_{n}$ (Theorem [5), using Theorem 4 together with some properties of the functions

$$
y_{n}={ }_{2} F_{1}\left(a+\varepsilon_{1} n, b+\varepsilon_{2} n ; c+\varepsilon_{3} n ; z\right),
$$

namely:

1. The three-term recurrence relations $f_{n+1}+\beta_{n} f_{n}+\alpha_{n} f_{n-1}=0$ have coefficients $\alpha_{n}$ and $\beta_{n}$ with finite limits as $n \rightarrow \infty$ (see Eq. (2.1) and (2.2)).

2. The coefficients $a_{n}, b_{n}, d_{n}, e_{n}$ of the difference-differential (DDE) equations

$$
\begin{aligned}
& f_{n}^{\prime}=a_{n} f_{n}+d_{n} f_{n-1}, \\
& f_{n-1}^{\prime}=b_{n} f_{n-1}+e_{n} f_{n},
\end{aligned}
$$

are of order $n$ as $n \rightarrow \infty$.

3. The coefficients $a_{n}, b_{n}, d_{n}, e_{n}, \alpha_{n}$ and $\beta_{n}$ are rational functions of $n$.

These properties have been verified for all the recurrences appearing in the present paper, that is for $\left|\epsilon_{j}\right| \leq 1, j=1,2,3$, and for the cases $\left(\epsilon_{1}, \epsilon_{2}, \epsilon_{3}\right)=$ $(1,0,2),(1,1,2),(1,1,3)(1,2,0)$, which, as we will see, appear when building the solutions for the basic recurrences $\left(\epsilon_{1}, \epsilon_{2}, \epsilon_{3}\right)=(1,1,0),(1,0,-1),(1,1,-1)$. Then, in a strict sense, Theorem 5 is only proved for these cases, although we conjecture that it is true in general for $\epsilon_{j} \in \mathbb{Z}$.

Theorem 5. Let $y_{n}={ }_{2} F_{1}\left(\begin{array}{c}a+\varepsilon_{1} n, b+\varepsilon_{2} n \\ c+\varepsilon_{3} n\end{array} ; z\right), \epsilon_{1}, \epsilon_{2}, \epsilon_{3} \in \mathbb{Z}$. For any $z \neq$ $0,1, \infty$ there exist $N>0$ and $C>0$ (independent of $n$ ), such that $\left|H_{n}^{\prime}(z)\right|<C$ for all $n>N$.

The same is true for $y_{n}=g(n) f(z)^{n}{ }_{2} F_{1}\left(\begin{array}{c}a+\varepsilon_{1} n, b+\varepsilon_{2} n \\ c+\varepsilon_{3} n\end{array} ;(z)\right), f(z)$ and $\zeta(z)$ being differentiable in $\mathbb{C} \backslash\{0,1, \infty\}$ and $g(n+1) / g(n)$ bounded for large $n$. 
Proof. The second part of the theorem follows immediately from the first part.

The functions $y_{n}={ }_{2} F_{1}\left(\begin{array}{c}a+\varepsilon_{1} n, b+\varepsilon_{2} n \\ c+\varepsilon_{3} n\end{array} ; z\right)$ satisfy DDEs (Eq. (2.6) $)$ and a TTRR. The coefficients of the TTRR and the DDE are related. Indeed, replacing $n$ by $n+1$ in the second equation in (2.6) and equating to the first equation we get the TTRR:

$$
f_{n+1}+\frac{b_{n+1}-a_{n}}{e_{n+1}} f_{n}-\frac{d_{n}}{e_{n+1}} f_{n-1}=0 .
$$

Therefore, given one of the solutions (for instance any of the 6 solutions we provide) of the TTRR $y_{n+1}+\beta_{n} y_{n}+\alpha_{n} y_{n-1}=0$, the coefficients of the system of DDEs satisfied by set of functions $\left\{y_{k}\right\}$ is related to the coefficients of the TTRR through:

$$
\begin{aligned}
& \beta_{n}=\frac{b_{n+1}-a_{n}}{e_{n+1}}=\frac{b_{n}-a_{n}}{e_{n}}(1+O(1 / n)), \\
& \alpha_{n}=-\frac{d_{n}}{e_{n+1}}=-\frac{d_{n}}{e_{n}}(1+O(1 / n)) .
\end{aligned}
$$

$H_{n}$, being a ratio of differentiable functions (except at the singular points), is differentiable. Using the DDEs we get:

$$
H_{n}^{\prime}=-e_{n}\left(H_{n}^{2}+\frac{b_{n}-a_{n}}{e_{n}} H_{n}-\frac{d_{n}}{e_{n}}\right)=-e_{n}\left(H_{n}^{2}+\beta_{n} H_{n}+\alpha_{n}+O(1 / n)\right) .
$$

Hence, because $\alpha_{n}=\alpha(1+O(1 / n))$ and $\beta_{n}=\beta(1+O(1 / n))$,

$$
H_{n}^{\prime}=-e_{n}\left[\left(H_{n}-t_{1}\right)\left(H_{n}-t_{2}\right)+O(1 / n)\right]
$$

Now, if $z$ is such that $\left|t_{1}\right| \neq\left|t_{2}\right|$, we have that either

$$
H_{n}=y_{n} / y_{n-1} \sim t_{1}(1+O(1 / n))
$$

or $H_{n} \sim t_{2}(1+O(1 / n))$ and then, because $e_{n}=O(n)$ then $H_{n}^{\prime}=O(1)$, which means that there exists $C>0$ such that $\left|H_{n}^{\prime}(z)\right|<C$ for large $n$.

Therefore, for large $n$ and when $\left|t_{1}\right| \neq\left|t_{2}\right|, H_{n}^{\prime}$ can only be singular on the singular points of the differential equation. Then, if for instance we have that, at $z=z_{0}$ (not a singular point), $\lim _{n \rightarrow \infty} H_{n}\left(z_{0}\right)=t_{1}\left(z_{0}\right)$ and $\left|t_{1}\left(z_{0}\right)\right| \neq\left|t_{2}\left(z_{0}\right)\right|$, this limit holds around $z_{0}$ and, in fact, it will be satisfied in any connected set not containing the singular points of the ODE or the curves $\left|t_{1}\right|=\left|t_{2}\right|$. This proves Theorem 3

Notice that, if $z$ is such that $\left|t_{1}\right|=\left|t_{2}\right|$, the argument is not true and the derivative may become unbounded as $n \rightarrow \infty$. This is consistent with the fact that the ratios $H_{n}$ may converge to $t_{1}$ at one side of a curve $\left|t_{1}\right|=\left|t_{2}\right|$ and to $t_{2}$ at the other side.

This theorem is crucial in simplifying our study because is states that a local analysis in the neighborhood of certain points (the singular points of the ODE) will suffice to obtain global information.

The second fundamental simplification comes from the fact that, as we will see, the curves $\left|t_{1}\right|=\left|t_{2}\right|$ divide the complex plane into disjoint regions such that in the interior of each of these disjoint regions there is only one singular point of the ODE. Besides, at each of these interior singular points, the characteristic roots behave differently, allowing for a simple identification of the minimal solution. Furthermore, we will see that for any of these interior singular points, the minimal 
solution around this point will be expressed in terms of an expansion around this same point.

Let us give an example for clarification. The recurrence relation for the case $(+++)$ (not taken as a basic case but related to $(00+))$ has as roots $t_{1}=1 /(1-z)$ and $t_{2}=-1 / z$. The curve $\left|t_{1}\right|=\left|t_{2}\right|$ is the vertical line $\Re z=1 / 2$, and there are two singular points $(z=0,1)$ that are away from this line, which we will call interior singular points. The minimal solution in the region $\Re z<1 / 2$ is an expansion around the interior singular point inside this region, namely:

$$
y_{n}={ }_{2} F_{1}\left(\begin{array}{c}
a+n, b+n \\
c+n
\end{array} ; z\right) .
$$

For proving this fact, by virtue of Theorem 3, we only need to know the behavior close to $z=0$, where $t_{1}$ and $t_{2}$ behave very differently.

Similarly, we can prove that the minimal solution for $\Re z>1 / 2$ can be expressed in terms of an expansion around the interior singular point in this region $(z=1)$, namely:

$$
y_{n}=(-1)^{n} \frac{\Gamma(c+n)}{\Gamma(1+a+b-c+n)}{ }_{2} F_{1}\left(\begin{array}{c}
a+n, b+n \\
1+a+b-c+n
\end{array} ; 1-z\right) .
$$

For proving this we only need to study the behaviour of the solution around $1-z=0$.

For studying the behaviour of the hypergeometric functions around $z=0$, the following result is enough:

Theorem 6. Let $y_{n}={ }_{2} F_{1}\left(\begin{array}{c}a+\epsilon_{1} n, b+\epsilon_{2} n \\ c+\epsilon_{3} n\end{array} ; z\right)$, with $\epsilon_{j}=0, \pm 1, \epsilon_{3} \neq 0, n=$ $0,1,2 \ldots$, and $c \notin \mathbb{Z}$ if $\epsilon_{3}<0$, then in a disc around the origin $\lim _{n \rightarrow \infty} \frac{y_{n}}{y_{n-1}}=t(z)$, where $t(z)$ is the root of the characteristic equation such that $\lim _{z \rightarrow 0} t(z)=1$.

This result is an immediate consequence of the following facts:

1. $H_{n}(0)=1$.

2. There exists $C>0$ such that $\left|H_{n}^{\prime}(0)\right|<C$ for all $n$. Indeed, it is easy to check that

$$
\left|H_{n}^{\prime}(0)\right| \leq\left|\frac{\epsilon_{1} \epsilon_{2}}{\epsilon_{3}}\right|+4\left|\frac{a b \epsilon_{3}^{2}+c^{2} \epsilon_{1} \epsilon_{2}-a c \epsilon_{2} \epsilon_{3}-b c \epsilon_{1} \epsilon_{3}}{\epsilon_{3}^{3}}\right| .
$$

3. One of the roots of the characteristic equation $(t(z))$ tends to 1 as $z \rightarrow 0$ while the other one goes to 0 or $\infty$. Both are continuous functions of $z$ in a disc around $z=0$ (excluding $z=0$ ) where the roots have different modulus.

The third condition is in fact a consequence of the fact that the characteristic roots are algebraic functions of $z$, that necessarily one of the roots tends to 1 as $z \rightarrow 1$ (because of the first condition) and that $\alpha(z)=\lim _{n \rightarrow \infty} \alpha_{n}$ either has a pole at $z=0$ or vanishes at $z=0$ (let us notice that the products of the characteristic roots is $\alpha(z))$. This condition has been tested explicitly for all the cases considered in this article. 


\section{ANAlysis OF THE FOUR BASIC CASES}

3.1. The $(++\mathbf{0})$ recursion. The recursion relation reads

$$
A(a+n, b+n) y_{n-1}+B(a+n, b+n) y_{n}+C(a+n, b+n) y_{n+1}=0,
$$

where

$$
\begin{aligned}
& A(a, b)=(c-a)(c-b)(c-a-b-1), \\
& B(a, b)=(c-a-b)\{c(a+b-c)+c-2 a b \\
& \quad+z[(a+b)(c-a-b)+2 a b+1-c]\}, \\
& C(a, b)=a b(c-a-b+1)(1-z)^{2} .
\end{aligned}
$$

The coefficients of characteristic equation $\lambda^{2}+\beta \lambda+\alpha=0$ are

$$
\alpha=1 /(1-z)^{2}, \quad \beta=-2(1+z) /(1-z)^{2}
$$

with roots

$$
t_{1}=\frac{1}{(1-\sqrt{z})^{2}}, \quad t_{2}=\frac{1}{(1+\sqrt{z})^{2}} .
$$

The equation $\left|t_{1}\right|=\left|t_{2}\right|$ holds when $z \leq 0$, otherwise $\left|t_{1}\right|>\left|t_{2}\right|$. In this case, the region $\left|t_{1}\right| \neq\left|t_{2}\right|$ is one connected region. The only singular point away from $\left|t_{1}\right|=\left|t_{2}\right|$ is $z=1$, and, as we will see, the minimal solution can be written in terms of an expansion around $z=1$ (both in the direction $n \rightarrow+\infty$ and $n \rightarrow-\infty$ ).

We provide six solutions of the recurrence relation using the method described in Section 1 .

$$
\begin{aligned}
& y_{1, n}={ }_{2} F_{1}\left(\begin{array}{c}
a+n, b+n \\
c
\end{array} z\right) \\
& y_{2, n}=\frac{\Gamma(1+a-c+n) \Gamma(1+b-c+n)}{\Gamma(a+n) \Gamma(b+n)}{ }_{2} F_{1}\left(\begin{array}{c}
1+a-c+n, 1+b-c+n \\
2-c
\end{array} ; z\right), \\
& y_{3, n}=\frac{\Gamma(1+a-c+n) \Gamma(1+b-c+n)}{\Gamma(1+a+b-c+2 n)}{ }_{2} F_{1}\left(\begin{array}{c}
a+n, b+n \\
1+a+b-c+2 n
\end{array} ; 1-z\right), \\
& y_{4, n}=(1-z)^{-2 n} \frac{\Gamma(a+b-c+2 n)}{\Gamma(a+n) \Gamma(b+n)}{ }_{2} F_{1}\left(\begin{array}{c}
-a+c-n,-b+c-n \\
1-a-b+c-2 n
\end{array} ; 1-z\right), \\
& y_{5, n}=(-z)^{-n} \frac{\Gamma(1+a-c+n)}{\Gamma(b+n)}{ }_{2} F_{1}\left(\begin{array}{c}
a+n, 1+a-c+n \\
1+\frac{1}{z}
\end{array}\right) \\
& y_{6, n}=(-z)^{-n} \frac{\Gamma(1+b-c+n)}{\Gamma(a+n)}{ }_{2} F_{1}\left(\begin{array}{c}
b+n, 1+b-c+n \\
1-a+b
\end{array}\right) .
\end{aligned}
$$


The minimal solution as $n \rightarrow \infty$ is $y_{3, n}$. Indeed, by Theorem 6 we have that, in a disc around $z=1$,

$$
\lim _{n \rightarrow+\infty} \frac{y_{3, n}}{y_{3, n-1}}=\frac{1}{4} t(\zeta), \quad \zeta=1-z .
$$

The factor $1 / 4$ comes from the limit of the ratio of gamma functions, while $t(\zeta)=$ $\lim _{n \rightarrow+\infty} y_{n} / y_{n-1}, y_{n} \equiv{ }_{2} F_{1}(a+n, b+n ; 1+a+b-c+2 n ; \zeta), \zeta=1-z$. According to Theorem [6, $\lim _{\zeta \rightarrow 0} t(\zeta)=1$.

The limit (3.5) corresponds to the root $t_{2}(z)$ of the $(++0)$ recurrence, because $t_{2}(z) \rightarrow 1 / 4$ as $z \rightarrow 1$. Also, because $t_{2}(z)$ is the smallest root outside the negative real axis, $y_{3, n}$ is minimal in a disc around $z=1$ and therefore, by Theorem 3 , is minimal in the complex plane except possibly on the negative real axis.

An explicit verification of the exact limit comes from the computation of $t(\zeta)$ for the $(++2+)$ recurrence relation. We indeed verify that

$$
\lim _{n \rightarrow+\infty} \frac{y_{3, n}}{y_{3, n-1}}=t_{2}(z), \quad z \in \mathbb{C} \backslash\{z \leq 0\} .
$$

Notice that the definition of $y_{3, n}$ indicates that the recurrence $(++0)$ and $(++2+)$ are in fact related.

Although it is not necessary to study the $(--0)$, because it can be related to the $(++0)$ by using the last relation in Eq. (1.4), it is also easy to obtain the minimal solution for the negative $n$ direction. The minimal solution in this case is $y_{4, n}$; indeed, from Theorems 3 and 6 we have that

$$
\lim _{n \rightarrow-\infty} \frac{y_{4, n}}{y_{4, n-1}}=\frac{4}{(1-z)^{2}} \frac{1}{t(\zeta)}=t_{1}(z), \quad z \in \mathbb{C} \backslash\{z \leq 0\} .
$$

All the six solutions solve the same recurrence relation, each of them can be expressed as a linear combination of two others with coefficients not depending on $n$. Any other solutions different from the minimal solution found cannot be minimal, because the minimal solution is unique (up to multiplicative factors not depending on $n$ ).

In summary, in $\mathbb{C} \backslash\{z \leq 0\}$ :

$$
\begin{array}{cc}
y_{1, n} & \text { dominant } \\
y_{2, n} & \text { dominant } \\
y_{3, n} & \text { minimal/dominant } \\
y_{4, n} & \text { dominant/minimal } \\
y_{5, n} & \text { dominant } \\
y_{6, n} & \text { dominant }
\end{array}
$$

where, when two possibilities appear, the first one corresponds to $n \rightarrow+\infty$ and the second one to $n \rightarrow-\infty$.

3.2. The $(\mathbf{0} 0+)$ recursion. The $(00+)$ recursion relation reads

$$
A(c+n) y_{n-1}+B(c+n) y_{n}+C(c+n) y_{n+1}=0,
$$


where

$$
\begin{aligned}
& A(c)=c(c-1)(z-1) \\
& B(c)=c[c-1-(2 c-a-b-1) z] \\
& C(c)=(c-a)(c-b) z
\end{aligned}
$$

The characteristic equation has coefficients $\alpha=1-1 / z$ and $\beta=-2+1 / z$. The characteristic roots are

$$
t_{1}=1, \quad t_{2}=\frac{z-1}{z}
$$

The curve $\left|t_{1}\right|=\left|t_{2}\right|$ is the straight line $\Re z=1 / 2$ and $\left|t_{1}\right|<\left|t_{2}\right|$ when $\Re z<1 / 2$. There are two interior singular points, that is, singular points which lie away from the curve $\left|t_{1}\right|=\left|t_{2}\right|: z=0,1$. Studying the solutions around these singular points will suffice for obtaining the minimal solutions at both sides of the line $\Re z=1 / 2$.

Solutions of the recurrence are:

$$
\begin{aligned}
& y_{1, n}={ }_{2} F_{1}\left(\begin{array}{c}
a, b \\
c+n
\end{array} ; z\right) \text {, } \\
& y_{2, n}=\left(\frac{z-1}{z}\right)^{n} \frac{\Gamma(-1+c+n) \Gamma(c+n)}{\Gamma(-b+c+n) \Gamma(-a+c+n)}{ }_{2} F_{1}\left(\begin{array}{c}
1-a, 1-b \\
2-c-n
\end{array} ; z\right) \text {, } \\
& y_{3, n}=\frac{\Gamma(c+n) \Gamma(-a-b+c+n)}{\Gamma(-b+c+n) \Gamma(-a+c+n)}{ }_{2} F_{1}\left(\begin{array}{c}
a, b \\
1+a+b-c-n
\end{array} ; 1-z\right) \text {, } \\
& y_{4, n}=\left(\frac{z-1}{z}\right)^{n} \frac{\Gamma(c+n)}{\Gamma(1-a-b+c+n)}{ }_{2} F_{1}\left(\begin{array}{c}
1-a, 1-b \\
1-a-b+c+n
\end{array} ; 1-z\right) \text {, } \\
& y_{5, n}=\frac{\Gamma(c+n)}{\Gamma(-a+c+n)}{ }_{2} F_{1}\left(\begin{array}{c}
a, 1+a-c-n \\
1+a-b
\end{array} ; \frac{1}{z}\right) \text {, } \\
& y_{6, n}=\frac{\Gamma(c+n)}{\Gamma(-b+c+n)}{ }_{2} F_{1}\left(\begin{array}{c}
b, 1+b-c-n \\
1-a+b
\end{array} ; \frac{1}{z}\right) \text {. }
\end{aligned}
$$

We have followed the method described in Section 1 and applied the last relation in Eq. (1.4) to the second and fourth solutions (in order to express the solutions in a more symmetrical form).

Proceeding as in the previous case (using Theorems [2, 3] and 6), it is clear that, when $\Re z<1 / 2$

$$
\lim _{n \rightarrow+\infty} \frac{y_{1, n}}{y_{1, n-1}}=t_{1}, \quad \lim _{n \rightarrow-\infty} \frac{y_{2, n}}{y_{2, n-1}}=t_{2} .
$$

Then, when $\Re z<1 / 2, y_{1, n}$ is minimal as $n \rightarrow+\infty$ and $y_{2, n}$ is minimal as $n \rightarrow-\infty$. 
Similarly, for $\Re z>1 / 2$ we may consider the expansions around the corresponding interior singular point. It is immediate to see that

$$
\lim _{n \rightarrow-\infty} \frac{y_{3, n}}{y_{3, n-1}}=t_{1}, \quad \lim _{n \rightarrow+\infty} \frac{y_{4, n}}{y_{4, n-1}}=t_{2},
$$

and because $\left|t_{1}\right|>\left|t_{2}\right|$ in this region, then $y_{4, n}$ is minimal as $n \rightarrow+\infty$ and $y_{3, n}$ is minimal as $n \rightarrow-\infty$.

Summarizing, we have:

$\begin{array}{ccc} & \Re z<\frac{1}{2} & \Re z>\frac{1}{2} \\ y_{1, n} & \text { minimal/dominant } & \text { dominant } \\ y_{2, n} & \text { dominant/minimal } & \text { dominant } \\ y_{3, n} & \text { dominant } & \text { dominant/minimal } \\ y_{4, n} & \text { dominant } & \text { minimal/dominant } \\ y_{5, n} & \text { dominant } & \text { dominant } \\ y_{6, n} & \text { dominant } & \text { dominant }\end{array}$

where, when two possibilities appear, the first one corresponds to $n \rightarrow+\infty$ and the second one to $n \rightarrow-\infty$.

It is important to realize that the last two solutions $y_{5, n}$ and $y_{6, n}$ are revealing that the recurrences $(00+)(+00)$ are related. There are several possible ways to express such a relation; for instance, by using the last relation in (1.4) and neglecting factors not depending on $n$, we can write the sixth solution:

$$
y_{6, n}(z)=\left(1-\frac{1}{z}\right)^{n} \frac{\Gamma(c+n)}{\Gamma(-b+c+n)}{ }_{2} F_{1}\left(\begin{array}{c}
1-a,-a+c+n \\
1-a+b
\end{array} ; \frac{1}{z}\right) .
$$

This means that, with some parameter substitutions and multiplying by some factors, we can transform a solution of the $(00+)$ recurrence (right side of the equation) into a solution of the $(++0)$ recurrence. The relation can also be inverted in order to build the solutions of the $(+00)$ relation from the solutions of the $(00+)$ relation. We postpone the analysis to Section 4

\subsection{The $(++-)$ recursion. The recursion relation reads}

$$
\begin{gathered}
A(a+n, b+n, c-n) y_{n-1}+B(a+n, b+n, c-n) y_{n} \\
+C(a+n, b+n, c-n) y_{n+1}=0,
\end{gathered}
$$

where

$$
\begin{aligned}
& A(a, b, c)=-(a-c)(a-c-1)(b-1-c)(b-c) z U, \\
& B(a, b, c)=c\left[c_{1} U+c_{2} V+c_{3} U V\right], \\
& c_{1}=(1-z)(b-c)(b-1)[a-1+z(b-c-1)], \\
& c_{2}=b(b+1-c)(1-z)(a+z(b-c+2)), \\
& c_{3}=c-2 b-(a-b) z \\
& C(a, b, c)=a b c(c-1)(1-z)^{3} V, \\
& U=z(a+b-c+1)(a+b-c+2)+a b(1-z), \\
& V=(1-z)(1-a-b+a b)+z(a+b-c-1)(a+b-c-2) .
\end{aligned}
$$


The coefficients of the characteristic equation are $\alpha=-16 z /(1-z)^{3}$ and $\beta=$ $\left(8 z^{2}+20 z-1\right) /(1-z)^{3}$. The zeros of the characteristic polynomial of the recursion relation (3.16) are

$$
t_{1}=\frac{32(1+w)}{(3+w)^{3}}, \quad t_{2}=\frac{32(1-w)}{(3-w)^{3}}, \quad w=\sqrt{8 z+1},
$$

where $w=\sqrt{8 z+1}$. Observe that $\lim _{z \rightarrow 0} t_{1}(z)=1$.

The curve $\left|t_{1}\right|=\left|t_{2}\right|$ in the complex $w$-plane is described by (we write $w=r e^{i \theta}$ )

$$
r=\sqrt{-9+6 \sqrt{3} \cos \theta}, \quad-\frac{1}{6} \pi \leq \theta \leq \frac{1}{6} \pi \quad \text { and } \quad \Re w=0,
$$

together with the half-line $z \leq-1 / 8$. This is shown in Figure 11. In the domain interior to the curve we have $\left|t_{1}\right|>\left|t_{2}\right|$.

There are two singular points for which $\left|t_{1}\right| \neq\left|t_{2}\right|$, which are $z=0,1$; on the other hand, $\lim _{z \rightarrow \infty} t_{1} / t_{2}=1$. As before, one can expect that the minimal solutions can be built by considering series around $z=0,1$.

We write the six solutions in the following way:

$$
\begin{aligned}
& y_{1, n}={ }_{2} F_{1}\left(\begin{array}{c}
a+n, b+n \\
c-n
\end{array} ; z\right), \\
& y_{2, n}=\left(\frac{z}{(z-1)^{3}}\right)^{n} \frac{\Gamma(b-c+1+2 n) \Gamma(a-c+1+2 n)}{\Gamma(a+n) \Gamma(b+n) \Gamma(1-c+n) \Gamma(2-c+n)} \\
& \times_{2} F_{1}\left(\begin{array}{c}
1-a-n, 1-b-n \\
2-c+n
\end{array} ; z\right), \\
& y_{3, n}=\frac{\Gamma(1+b-c+2 n) \Gamma(1+a-c+2 n)}{\Gamma(1-c+n) \Gamma(1+a+b-c+3 n)}{ }_{2} F_{1}\left(\begin{array}{c}
a+n, b+n \\
1+a+b-c+3 n
\end{array} ; 1-z\right) \text {, } \\
& y_{4, n}=\left(\frac{z}{(z-1)^{3}}\right)^{n} \frac{\Gamma(a+b-c+3 n)}{\Gamma(a+n) \Gamma(b+n) \Gamma(1-c+n)} \\
& \times_{2} F_{1}\left(\begin{array}{c}
1-a-n, 1-b-n \\
1-a-b+c-3 n
\end{array} ; 1-z\right), \\
& y_{5, n}=z^{-n} \frac{\Gamma(1+a-c+2 n)}{\Gamma(b+n) \Gamma(1-c+n)}{ }_{2} F_{1}\left(\begin{array}{c}
a+n, 1+a-c+2 n \\
1+a-b
\end{array} ; \frac{1}{z}\right) \text {, } \\
& y_{6, n}=z^{-n} \frac{\Gamma(1+b-c+2 n)}{\Gamma(n+a) \Gamma(1-c+n)}{ }_{2} F_{1}\left(\begin{array}{c}
b+n, 1+b-c+2 n \\
1+b-a
\end{array} ; \frac{1}{z}\right) \text {. }
\end{aligned}
$$

Proceeding like in the previous cases, it is evident that inside the curve

$$
\lim _{n \rightarrow-\infty} \frac{y_{1, n}}{y_{1, n-1}}=t_{1}
$$

and therefore $y_{1, n}$ is minimal as $n \rightarrow-\infty$ because $\left|t_{1}\right|>\left|t_{2}\right|$, whereas

$$
\lim _{n \rightarrow+\infty} \frac{y_{2, n}}{y_{2, n-1}}=\frac{16 z}{(z-1)^{3}} \frac{1}{t_{1}}=\frac{\alpha}{t_{1}}=t_{2},
$$

and hence $y_{2, n}$ is minimal as $n \rightarrow+\infty$. 


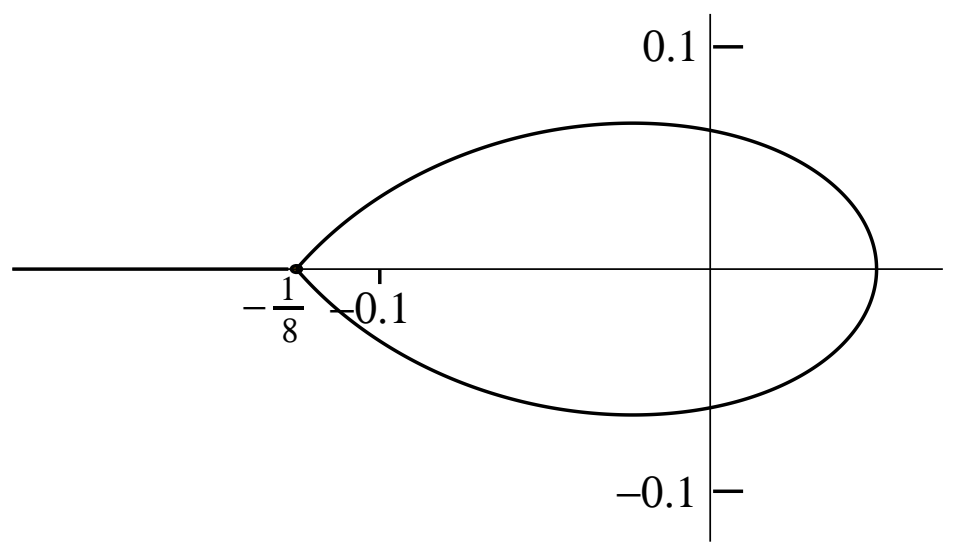

Figure 1. The curve $\left|t_{1}\right|=\left|t_{2}\right|$ for the case $(++-)$.

Outside the curve, we have to consider the series around $z=1$, that is, the solutions $y_{3, n}$ and $y_{4, n}$. We have that

$$
\lim _{n \rightarrow+\infty} \frac{y_{3, n}}{y_{3, n-1}}=\frac{16}{27} t(\zeta), \quad \zeta=1-z,
$$

where $t(\zeta)$ is the characteristic root for the TTRR satisfied by

$$
y_{n}={ }_{2} F_{1}\left(\begin{array}{c}
a+n, b+n \\
c+3 n
\end{array} ; \zeta\right),
$$

such that $\lim _{\zeta \rightarrow 0} t(\zeta)=1$. Therefore, because $\lim _{z \rightarrow 1} t_{1}(z) \rightarrow \frac{16}{27}$ (whereas $t_{2}$ is singular at $z=1$ ) it is clear that

$$
\lim _{n \rightarrow+\infty} \frac{y_{3, n}}{y_{3, n-1}}=t_{1}
$$

and therefore $y_{3, n}$ is minimal as $n \rightarrow+\infty$. The limit can be explicitly checked by computing $t(z)$; for brevity, we don't provide the details of such a calculation.

On the other hand

$$
\lim _{n \rightarrow-\infty} \frac{y_{4, n}}{y_{4, n-1}}=\frac{27 z}{(z-1)^{3}} \frac{1}{t(\zeta)}=\frac{27 \alpha}{16 t(\zeta)}=t_{2},
$$

and hence $y_{4, n}$ is minimal as $n \rightarrow-\infty$.

Summarizing:

$\begin{array}{ccc} & \text { inside the curve } & \text { outside the curve } \\ y_{1, n} & \text { dominant/minimal } & \text { dominant } \\ y_{2, n} & \text { minimal/dominant } & \text { dominant } \\ y_{3, n} & \text { dominant } & \text { minimal/dominant } \\ y_{4, n} & \text { dominant } & \text { dominant/minimal } \\ y_{5, n} & \text { dominant } & \text { dominant } \\ y_{6, n} & \text { dominant } & \text { dominant }\end{array}$

where, when two possibilities appear, the first one corresponds to $n \rightarrow+\infty$ and the second one to $n \rightarrow-\infty$. 
Observe that the above results only hold inside the principal sector where $-\pi<$ phase $(8 z+1)<\pi$, that is, in the cut plane, with branch cut from $-\frac{1}{8}$ to $-\infty$.

3.4. The $(+0-)$ recursion. The $(+0-)$ recursion relation reads

$$
A(a+n, c-n) y_{n-1}+B(a+n, c-n) y_{n}+C(a+n, c-n) y_{n+1}=0,
$$

where

$$
\begin{aligned}
A(a, c) & =z(a-c)(a-c-1)(b-c)[a+z(b+1-c)] \\
B(a, c) & =c[a(a-1)(c-1)+a(a-1)(a+3 b-4 c+2) z \\
& \left.+(b-c)(b+1-c)(4 a-c-1) z^{2}-(a-b)(b-c)(b+1-c) z^{3}\right], \\
C(a, c) & =-a c(c-1)[a-1+z(b-c)](1-z)^{2} .
\end{aligned}
$$

The coefficients of the characteristic equation are $\alpha=-4 z /(1-z)^{2}$ and $\beta=$ $-\left(z^{2}-6 z+1\right) /(1-z)^{2}$. The zeros of the characteristic polynomial of the recursion relation (3.27) are

$$
t_{1}=1, \quad t_{2}=-\frac{4 z}{(1-z)^{2}} .
$$

The curve defined by $\left|t_{1}\right|=\left|t_{2}\right|$ is described by

$$
r=2+\cos \theta \pm \sqrt{\cos ^{2} \theta+4 \cos \theta+3}, \quad-\pi \leq \theta \leq \pi, \quad z=r e^{i \theta} .
$$

Both signs give a closed loop with common point -1. In Figure 2 we show this curve in the $z$-plane. In the domain interior to the inner curve we have $\left|t_{1}\right|>\left|t_{2}\right|$; between the inner curve and the outer curve we have $\left|t_{1}\right|<\left|t_{2}\right|$, and outside the outer curve $\left|t_{1}\right|>\left|t_{2}\right|$.

It is important to notice that the three singular points $z=0,1, \infty$ are interior in this case, each one inside a different region of the three regions into which the plane is divided. Then, expansions around each of the singular points will be needed in order to identify the minimal solution in each region.

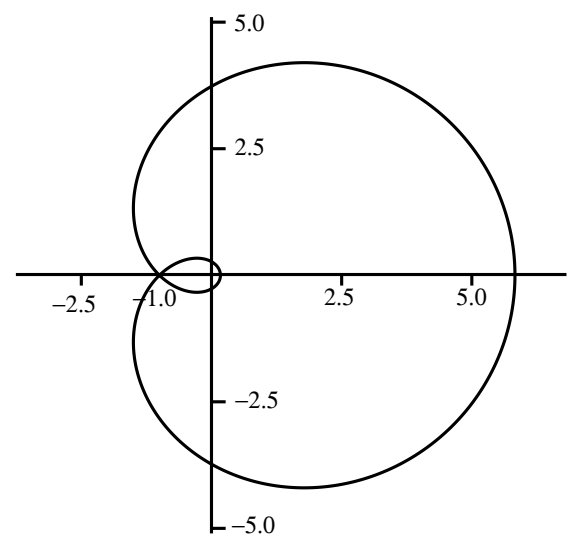

Figure 2. The curve $\left|t_{1}\right|=\left|t_{2}\right|$ for the $(+0-)$ recursion. 
The six solutions for this case can be written:

$$
\begin{aligned}
y_{1, n}= & { }_{2} F_{1}\left(\begin{array}{c}
a+n, b \\
c-n
\end{array} ;\right), \\
y_{2, n}= & \left(\frac{-z}{(1-z)^{2}}\right)^{n} \frac{\Gamma(1+b-c+n) \Gamma(1+a-c+2 n)}{\Gamma(a+n) \Gamma(1-c+n) \Gamma(2-c+n)} \\
& \quad \times_{2} F_{1}\left(\begin{array}{c}
1-a-n, 1-b \\
2-c+n
\end{array} ; z\right), \\
y_{3, n}= & \frac{\Gamma(1+b-c+n) \Gamma(1+a-c+2 n)}{\Gamma(1-c+n) \Gamma(1+a+b-c+2 n)}{ }_{2} F_{1}\left(\begin{array}{c}
a+n, b \\
1+a+b-c+2 n
\end{array} ; 1-z\right), \\
y_{4, n}= & \left(\frac{-z}{(1-z)^{2}}\right)^{n} \frac{\Gamma(a+b-c+2 n)}{\Gamma(a+n) \Gamma(1-c+n)} \\
& \quad \times{ }_{2} F_{1}\left(\begin{array}{c}
1-a-n, 1-b \\
1-a-b+c-2 n
\end{array} ; 1-z\right), \\
y_{5, n}= & \left(\frac{-z}{(1-z)^{2}}\right)^{n} \frac{\Gamma(1+a-c+2 n)}{\Gamma(1-c+n) \Gamma(1+a-b+n)}{ }_{2} F_{1}\left(\begin{array}{c}
1-b,-b+c-n \\
1+a-b+n
\end{array} ; \frac{1}{z}\right), \\
y_{6, n}= & \frac{\Gamma(a-b+n) \Gamma(1+b-c+n)}{\Gamma(a+n) \Gamma(1-c+n)}{ }_{2} F_{1}\left(\begin{array}{c}
b, 1+b-c+n \\
1-a+b-n
\end{array} ; \frac{1}{z}\right) .
\end{aligned}
$$

The situation, as we next prove, can be summarized in the following way:

$\begin{array}{lc} & \text { inside inner curve } \\ y_{1} & \text { dominant/minimal } \\ y_{2} & \text { minimal/dominant } \\ y_{3} & \text { dominant } \\ y_{4} & \text { dominant } \\ y_{5} & \text { dominant } \\ y_{6} & \text { dominant }\end{array}$

between curves
dominant
dominant
minimal/dominant
dominant/minimal
dominant
dominant

outside outer curve dominant dominant dominant dominant minimal/dominant dominant/minimal

where, when two possibilities appear, the first one corresponds to $n \rightarrow+\infty$ and the second one to $n \rightarrow-\infty$.

Let us now search for the minimal solutions.

3.4.1. Minimal solutions inside the inner curve. This region contains the point $z=$ 0 , and we should consider expansions around this point. In this region $\left|t_{2}\right|<\left|t_{1}\right|$.

Proceeding as before, it is obvious that

$$
\lim _{n \rightarrow-\infty} \frac{y_{1, n}}{y_{1, n-1}}=t_{1} .
$$

Therefore, $y_{1, n}$ is minimal as $n \rightarrow-\infty$.

On the other hand

$$
\lim _{n \rightarrow+\infty} \frac{y_{2, n}}{y_{2, n-1}}=\frac{-4 z}{(1-z)^{2}} \frac{1}{t_{1}}=\frac{\alpha}{t_{1}}=t_{2}
$$

and hence $y_{2, n}$ is minimal as $n \rightarrow+\infty$. 
3.4.2. Minimal solutions between curves. For the $y_{3, n}$ solution, we have:

$$
\lim _{n \rightarrow+\infty} \frac{y_{3, n}}{y_{3, n-1}}=t(\zeta)
$$

where $\zeta=1-z$ and $t(\zeta)$ is the characteristic root for the TTRR satisfied by

$$
y_{n}={ }_{2} F_{1}\left(\begin{array}{c}
a+n, b \\
c+2 n
\end{array} ; \zeta\right),
$$

such that $\lim _{\zeta \rightarrow 0} t(\zeta)=1$. Therefore, because $t_{1}(z)=1$ (while $t_{2}$ is singular at $z=1)$ it is clear that

$$
\lim _{n \rightarrow+\infty} \frac{y_{3, n}}{y_{3, n-1}}=t_{1}
$$

and therefore $y_{3, n}$ is minimal as $n \rightarrow+\infty$. The limit can be explicitly checked by computing $t(\zeta)$, which is equal to $t_{1}=1$.

On the other hand

$$
\lim _{n \rightarrow-\infty} \frac{y_{4, n}}{y_{4, n-1}}=\frac{\alpha(z)}{t(\zeta)}=t_{2},
$$

and therefore $y_{4, n}$ is minimal as $n \rightarrow-\infty$.

3.4.3. Minimal solutions outside the outer curve. In this region we have the singular point $z=\infty$, and we consider a series around this point. We have

$$
\lim _{n \rightarrow+\infty} \frac{y_{5, n}}{y_{5, n-1}}=\frac{\alpha}{t_{1}}=t_{2}
$$

and

$$
\lim _{n \rightarrow-\infty} \frac{y_{6, n}}{y_{6, n-1}}=t_{1} .
$$

Therefore $y_{5, n}$ is minimal as $n \rightarrow+\infty$ while $y_{6, n}$ is minimal as $n \rightarrow-\infty$.

\section{Obtaining INFORMation FOR the REMAining $22+4$ RECURRENCES}

As discussed in [3], the 26 recurrence relations with $\left|\epsilon_{j}\right| \leq 1$ can be reduced to 5 cases by means of Eq. (1.4). In addition, as we discussed before, when building the six solutions by considering linear relations between solutions, new relations appear, as is the case of the relation between the $(00+)$ recurrence and the $(+00)$; this relation reduces the number of basic recurrence relations to 4 . We also saw that 4 other recurrences are related with the 4 remaining basic recurrences: $(++2+)$ with $(++0),(++3+)$ and $(+2+0)$ with $(++-)$, and $(+02+)$ with $(+0-)$.

All these cases can be related to the four basic cases by using simple transformations.

Let us for instance consider obtaining all the information for the $(+00)$ from the $(00+)$ recurrence. From Eq. (3.15) we see that the solutions $y_{\alpha, \beta ; \gamma+n}^{(00+)}(z)$ of the recurrence satisfied by the hypergeometric functions ${ }_{2} F_{1}(\alpha, \beta ; \gamma+n ; z)$ can be built from the solutions $y_{\alpha+n, \beta ; \gamma}^{(+00)}(z)$ of the recurrence satisfied by the functions ${ }_{2} F_{1}(\alpha+n, \beta ; \gamma ; z)$ by means of the following transformation:

$$
y_{\alpha, \beta ; \gamma+n}^{(00+)}(z)=\left(1-\frac{1}{z}\right)^{n} \frac{\Gamma(\gamma+n)}{\Gamma(-\beta+\gamma+n)} y_{-\alpha+\gamma+n, 1-\alpha ; 1-\alpha+\beta}^{(+00)}(1 / z) .
$$


$y_{\alpha, \beta ; \gamma+n}^{(00+)}(z)$ denotes a generic solution of the $(00+)$ recurrence, and not only the ${ }_{2} F_{1}(\alpha, \beta ; \gamma+n ; z)$ function; also $y_{\alpha+n, \beta ; \gamma}^{(+00)}(z)$ is a generic solution of the $(+00)$ recurrence. Notice that the relation (3.15) provides not only a connection between two Gauss hypergeometric functions, but also a transformation of a recurrence into another one $((+00)$ into $(00+))$. This transformation can be considered for any solution (in particular for the six solutions given for each recurrence).

Denoting $a=-\alpha+\gamma, b=1-\alpha, c=1-\alpha+\beta$, we can invert this general relation to get:

$$
y_{a+n, b ; c}^{(+00)}(z)=(1-z)^{-n} \frac{\Gamma(1+a-c+n)}{\Gamma(1+a-b+n)} y_{1-b,-b+c ; 1+a-b+n}^{(00+)}(1 / z) .
$$

Therefore, we can build the six solutions for the $(+00)$ recurrence by performing the following transformations over the solutions of the $(00+)$ recurrence:

1. Perform the substitutions: $a \rightarrow 1-b, b \rightarrow-b+c, c \rightarrow 1+a-b, z \rightarrow 1 / z$.

2. Multiply the resulting functions by $(1-z)^{-n} \frac{\Gamma(1+a-c+n)}{\Gamma(1+a-b+n)}$.

With these transformations, we get the following set of solutions for the $(+00)$ case:

$$
\begin{aligned}
& y_{1, n}=(1-z)^{-n} \frac{\Gamma(1+a-c+n)}{\Gamma(1+a-b+n)}{ }_{2} F_{1}\left(\begin{array}{c}
\left.1-b,-b+c ; \frac{1}{z}\right), \\
1+a-b+n
\end{array}\right) \\
& y_{2, n}=\frac{\Gamma(a-b+n)}{\Gamma(a+n)}{ }_{2} F_{1}\left(\begin{array}{c}
b, 1+b-c \\
1-a+b-n
\end{array} ; \frac{1}{z}\right), \\
& y_{3, n}=(1-z)^{-n} \frac{\Gamma(a+b-c+n)}{\Gamma(a+n)}{ }_{2} F_{1}\left(\begin{array}{c}
-a+c-n,-b+c \\
1-a-b+c-n
\end{array} ; 1-z\right), \\
& y_{4, n}=\frac{\Gamma(1+a-c+n)}{\Gamma(1+a+b-c+n)}{ }_{2} F_{1}\left(\begin{array}{c}
a+n, b \\
1+a+b-c+n
\end{array} ; 1-z\right), \\
& y_{5, n}=\frac{\Gamma(1+a-c+n)}{\Gamma(a+n)}{ }_{2} F_{1}\left(\begin{array}{c}
1+a-c+n, 1+b-c ; z \\
2-c
\end{array}\right), \\
& y_{6, n}={ }_{2} F_{1}\left(\begin{array}{c}
a+n, b \\
c
\end{array}\right) .
\end{aligned}
$$

Here, apart from the prescribed substitutions, the last transformation of Eq. (1.4) has also been applied in the last 4 cases (and the factors not depending on $n$ removed).

Given that we make the substitution $z \rightarrow 1 / z$ and we multiply the solutions by $\left(\frac{1}{1-z}\right)^{n}$, the characteristic roots are the roots for $(00+)$ with $z$ replaced by $1 / z$ and then multiplied by $1 /(1-z)$, that is

$$
t_{1}=\frac{1}{1-z}, t_{2}=1
$$


Of course, the character of the solutions is maintained in the transformed domains given by the change $z \rightarrow 1 / z$. We therefore have:

$\begin{array}{ccc} & \Re\left(z^{-1}\right)<\frac{1}{2} & \Re\left(z^{-1}\right)>\frac{1}{2} \\ y_{1, n} & \text { minimal/dominant } & \text { dominant } \\ y_{2, n} & \text { dominant/minimal } & \text { dominant } \\ y_{3, n} & \text { dominant } & \text { dominant/minimal } \\ y_{4, n} & \text { dominant } & \text { minimal } / \text { dominant } \\ y_{5, n} & \text { dominant } & \text { dominant } \\ y_{6, n} & \text { dominant } & \text { dominant }\end{array}$

Observe that the equation $\Re\left(z^{-1}\right)=1 / 2$ is the equation of the circle $|1-z|=1$, with $\Re\left(z^{-1}\right)>\frac{1}{2}$ defining the interior of the disk and $\Re\left(z^{-1}\right)<\frac{1}{2}$ the exterior.

For this transformation chosen we have the most general situation possible: the parameters and the variable are replaced, and the functions are multiplied by gamma functions and by a $z$-dependent factor. A more simple example is provided by the connection of $(00+)$ with the $(+++)$ case. Using the last relation in Eq. (1.4), we have:

$$
{ }_{2} F_{1}\left(\begin{array}{c}
a+n, b+n \\
c+n
\end{array} ; z\right)=(1-z)^{c-a-b-n}{ }_{2} F_{1}\left(\begin{array}{c}
c-a, c-b \\
c+n
\end{array} ; z\right) .
$$

Therefore, the solutions of the $(+++)$ recurrence can be obtained from the solutions of the $(00+)$ by multiplying by $(1-z)^{-n}$ and by substituting $a$ by $c-a$ and $b$ by $c-b$.

\section{Conclusions}

The problem of obtaining the pairs of satisfactory numerical solutions of the hypergeometric recursions satisfied by the functions $y_{n}={ }_{2} F_{1}\left(\begin{array}{c}a+\epsilon_{1} n, b+\epsilon_{2} n \\ c+\epsilon_{3}\end{array} ; z\right)$, $\left|\epsilon_{j}\right| \leq 1$ has been solved in the complex plane, except on the critical curves $\left|t_{1}\right|=\left|t_{2}\right|$ $\left(t_{1}\right.$ and $t_{2}$ being the roots of the characteristic polynomial) where the Poincaré theorem does not provide information regarding the existence of minimal solutions.

The study of the behaviour on the critical curves needs a separate analysis and is beyond the scope of the present paper. In [2], the real case for the recurrence $(+++)$ is discussed in detail. In this case it is easy to check that for $z=1 / 2$ there is a minimal solution when $1+a+b-2 c \neq 0$; this fact has important consequences on the stability of the recurrences close to $z=1 / 2$.

A comprehensive description of the condition of the hypergeometric recursions in the complex plane is now available, except on the critical curves. This is an essential piece of information for the computation of hypergeometric functions by means of recurrence relations.

\section{ACKNOWLEDGMENTS}

The authors wish to thank the referee for comments and suggestions which have resulted in an improved version of the paper. A. Gil acknowledges financial support from Ministerio de Educación y Ciencia (programa Ramón y Cajal). J. Segura acknowledges financial support from project BFM2003-06335-C03-02 and from the 
Secretaría de Estado de Universidades e Investigación (Programa de Movilidad, Ref. PR2005-0333). The authors acknowledge financial support from Ministerio de Educación y Ciencia (Project MTM2004-01367).

\section{REFERENCES}

[1] M. Abramowitz, I. Stegun (Eds). Handbook of Mathematical Functions. National Bureau of Standards. Applied Mathematics Series, no. 55. U.S. Government Printing Office, Washington DC (1964). MR0167642 (29:4914)

[2] A. Deaño, J. Segura. Transitory minimal solutions of hypergeometric recursions and pseudoconvergence of associated continued fractions. Accepted for publication in Mathematics of Computation.

[3] A. Gil, J. Segura, N. M. Temme. The ABC of hyper recursions. J. Comput. Appl. Math.

[4] Y. L. Luke. The special functions and their approximations, Vol. I. Mathematics in Science and Engineering, Vol. 53., Academic Press, New York, 1969. MR0241700 (39:3039)

[5] G.N. Watson. Asymptotic expansions of hypergeometric functions. Trans. Cambridge Philos. Soc., 22:277-308, 1918.

[6] J. Wimp. Computation with recurrence relations. Applicable Mathematics Series. Pitman (Advanced Publishing Program). Boston, MA. 1984. MR0727118 (85f:65001)

Departamento de Matemáticas, Estadística y Computación, Univ. Cantabria, 39005SANTANDER, SPAIN

E-mail address: amparo.gil@unican.es

Departamento de Matemáticas, Estadística y Computación, Univ. Cantabria, 39005SANTANDER, SPAin

E-mail address: javier.segura@unican.es

CWI, P.O. Box 94079, 1090 GB Amsterdam, The Netherlands

E-mail address: nicot@cwi.nl 